\title{
Intelligent Assembly/Disassembly System with a Haptic Device for Aircraft Parts Maintenance
}

\author{
Christiand and Jungwon Yoon* \\ Mechanical and Aerospace Engineering and ReCAPT, \\ GyeongSang National University, Jinju, Gyeongnam 660-701, Korea \\ tianize@yahoo.com, jwyoon@gnu.ac.kr
}

\begin{abstract}
This paper presents the development of an intelligent Assembly/Disassembly (A/D) system with the utilization of a haptic device. The development was aimed to assist the A/D process of aircraft parts maintenance with an intelligent algorithm. A comprehensive methodology is presented by an intelligent algorithm generating the best sequence for the assembled/disassembled parts. For this purpose, a genetic algorithm was chosen because of its effectiveness and compatibility dealing with the A/D sequencing problem. Furthermore, A/D process of the parts will also be calculated in the genetic algorithm process, which will show the effectiveness of the algorithm. Moreover, the concept of both natural and artificial constraints are applied to generate the path planning of the assembled/disassembled parts. It is believed that the combination of haptic, optimized sequence algorithm and intelligent path planning will increase efficiency for the overall aircraft parts maintenance process.
\end{abstract}

Keywords: Haptic, Genetic Algorithm, Natural and Artificial Constraint, Aircraft Part Maintenance.

\section{Introduction}

The implementation of Virtual Reality (VR) technologies such as the medical and $\mathrm{A} / \mathrm{D}$ fields, is increasing. The improvement of VR technologies promises a bright future for the efficiency of the A/D process, including the maintenance of aircraft parts. Since the physical objects are represented in digital form with VR technologies, users do not need to have a real object for the simulation of the process. From that point of view, the efficiency can be increased because of the elimination of real object. Furthermore, the development of VR technologies is also supported by the development of haptic technologies. Nowadays, some haptic devices are available and widely used such as Phantom families [1] from SenseAble ${ }^{\mathrm{TM}}$ Corporation and Omega $\mathrm{x}^{\mathrm{TM}}$ from the Force Dimension Corporation. Haptic devices give touch sensing by a force-feedback mechanism. Through this mechanism, an operator will feel the result of collision force.

\footnotetext{
* Corresponding authors.
} 
The maintenance process itself benefits from advancements in the VR interface, since the maintenance process involves many A/D task and depending on the amount of parts involved. A maintenance simulation could be held to observe the scheme of maintenance task by simulating the process in a virtual environment (VE). Borro et al. have developed the system for the maintainability simulation in aeronautics. They combined a haptic device to the system and keep the user's movements same as those that occur when testing a physical mock-up [2]. Similar work has also been suggested by Saval et al. [3]. Another research focused on the development a language, such as Virtual Fixture Assembly Language (VFAL) [4]. This library could be used to construct various virtual fixture series with graphic and force guidance rules, making the low-level haptic and graphic rendering details transparent to the developers. Gallantuci et. al have researched the A/D planning by using fuzzy and genetic algorithms [5]. They utilized a genetic algorithm (GAs) to generate the optimal sequence for A/D task.

Our research focuses on the development of A/D system for the aircraft part maintenance process. We propose a combination of the utilization of a haptic device and an intelligent algorithm for part sequence generation. We utilized a genetic algorithm as an optimization method to optimize part sequence generation. Even though some researches already have been suggested this for the parts sequence generation, they didn't consider the utilization of a haptic device. Natural and artificial constraint concepts are applied in this paper. Natural and artificial constraint concepts have been widely applied in the robotics field. Even though there has been extensive research [6-7] on the constraints in the haptics field, there has not been a detailed study on its applicability to aircraft part maintenance. This paper describes the development of the intelligent system for the aircraft parts maintenance with haptic information.

\section{Description of Aircraft Part Maintenance System}

The schematic of A/D system is shown in Figure 1. The system consists of a haptic device (Phantom omni), a Virtual Environment (VE), and a trained operator. The haptic device can be considered as an apparatus where an operator inputs the action for the manipulated object. This device has only three degree of freedom (3-DOF), so it can not generate torque. The communication occurred bi-directionally between the haptic device and the VE through a fire-wire cable. The manipulated objects are aircraft parts with a scale model, which was made in CAD software and saved as the VRML (Virtual Reality Machine Language) file format. After the object was transferred to VE, the operator could then manipulate the object during the scheme of the maintenance task.

In the future trials according to the design, the movement made by the operator will be constrained along to the suggested path. As the operator looks at VE, he/she will feel the guidance force from artificial and natural constraints. The artificial and natural constraints will guide the operator to put the part in the right position, using the suggested path. The virtual environment was built based on the OpenInventor ${ }^{\mathrm{TM}}$ graphic library, a collision detection library, a path planning algorithm, and an optimal sequence generation algorithm. Those components work together to make the aircraft part maintenance system efficient. 


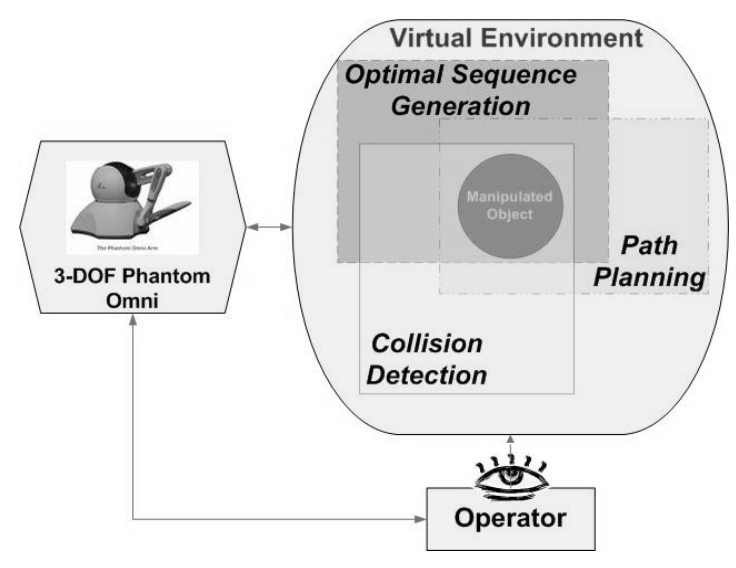

(a) Schematic of A/D System

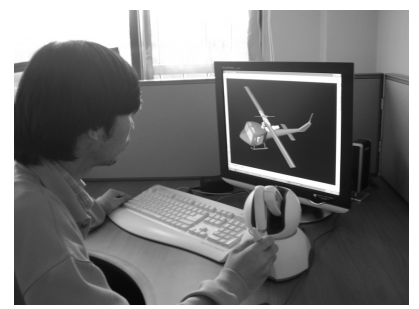

(b) An operator

Fig. 1. An intelligent $A / D$ system for aircraft parts maintenance

\section{Aircraft Maintenance System Algorithm}

The overall system algorithm is shown in the flowchart in Fig. 2. First of all, the system should check the haptic device through the device initialization, verify the initial position of a haptic stylus, and then confirm whether collision detection is being performed or not. This step was aimed to decide which part will be the first position in the sequence. This procedure gives freedom to the operator to set the first suitable part as he/she wish. Since the collision detection library only retrieves the coordinates of the colliding polygons and points, the contact configuration needs to be reconstructed by considering the possible combination of surface features between two bodies [6]. For collision detection, the functions from graphic API (Application Programming Interface) Open Inventor are utilized [8].

After the first part of the algorithm is recognized, we can perform the optimal sequence generation. The genetic algorithm (GAs) is applied in this step. The GAs algorithm begins by defining the optimization variables, the cost function, and the cost [9]. The cost function is a function that shows the relation between the optimization variable and the parameter for evaluation of the problem solution. The cost is a difference between the actual solution and the desired solution. Optimization variables are the variables that should be optimized to reach optimal solution for the case model. The optimization variables are arranged in chromosome form. A cluster of chromosome is called as a population. The optimization variables are sequence order $(\mathrm{S})$, direction of each part $(\mathrm{O})$, and used tool for each part $(\mathrm{T})$.

$$
\text { chromosome }=[S|O| T]
$$

where : 
$S=\left(s_{1} s_{s} \cdots s_{n}\right)=$ part sequence

$O=\left(o_{1} o_{s} \cdots o_{n}\right)=$ part orientation

$T=\left(t_{1} t_{s} \cdots t_{n}\right)=$ used tool for each part

$n=$ number of part

The evaluation of disassembly sequence was realized by using cost function [5]:

Cost function $=f($ chromosome $)=\left(w_{1} \cdot l\right)+w_{2} \cdot(N-1-o)+w_{3} \cdot(N-g-1)$

where the parameters of the cost function were set to $1, \mathrm{o}$, and $\mathrm{g}$ that variables are the maximum length of feasible sequence, the orientation change number, the gripper change number, respectively. Then, every parameter was associated with the weight $w$.

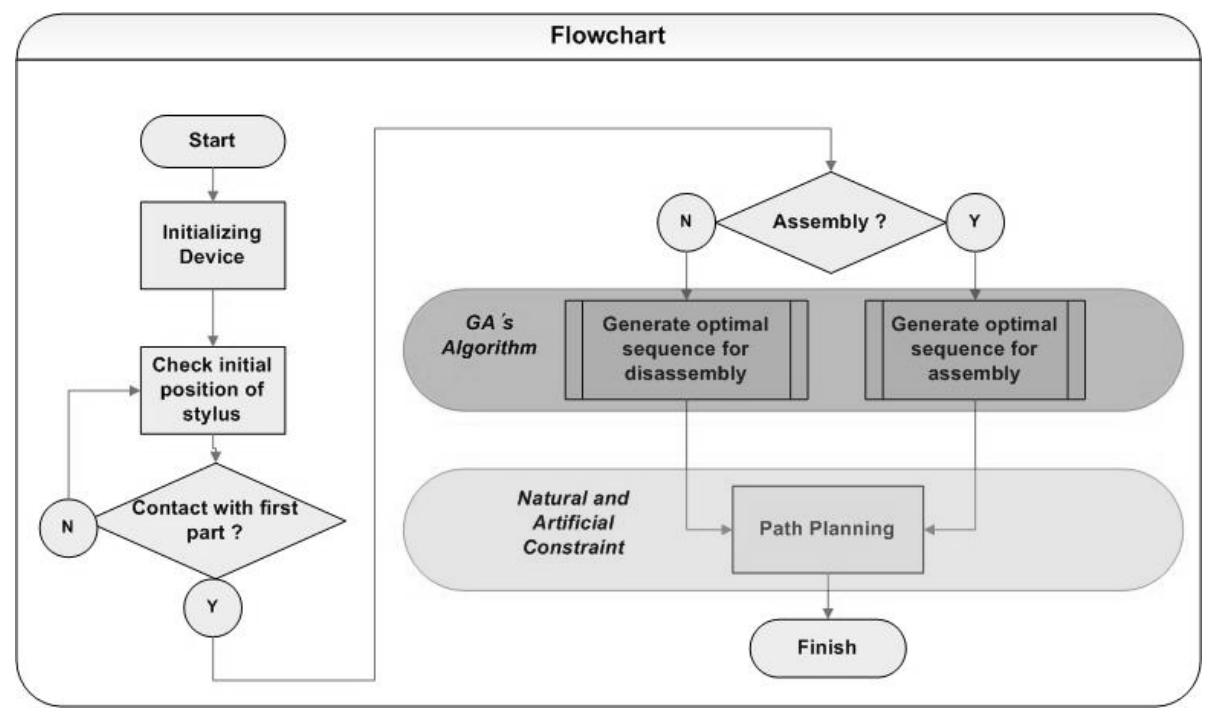

Fig. 2. System algorithm of the intelligent A/D system

The use of these cost function parameters are quite representative for the case such as the maintenance task. In the maintenance task, the numbers of orientation changes and tool changes will affect the efficiency of overall maintenance process. An analogy can be made to describe the effect of the cost function parameters for the overall efficiency. A gripper is a tool used to do one task of the A/D tasks, whereas the orientation changes represent how many step we needed for doing one task in A/D task list. It needs to be restated in this case, that the best sequence is a list of parts in the assembled order which have the highest cost function value.

The ranking for chromosomes will be generated in every generation (iteration). Ranking is made by sorting the cost value for each chromosome. Some portion of population will be destroyed to give space for new offspring. This process is called 
natural selection. Selection rate $\left(X_{\text {rate }}\right)$ determines the amount of chromosomes that still exists after the natural selection process. The selection rate is set to $50 \%$. The relation between the existing chromosomes $\left(P_{\text {keep }}\right)$ and the number of population $(P)$ is shown in (3).

$$
P_{\text {keep }}=X_{\text {rate }} \times P
$$

Then, the numbers of the empty space or the disappearing chromosomes are shown in (4).

$$
P_{\text {disappear }}=P-P_{\text {keep }}
$$

Half of the population will disappear and will be replaced by new offspring. The disappeared chromosomes $\left(P_{\text {disappear }}\right)$ are chromosomes that have low cost values. In each generation, GAs operator: crossover and mutation are applied to produce new offspring. By applying the GAs operator, the fitness of the chromosomes can be increased. Crossover is a process matching of some nodes in one chromosome to another chromosome. The results of the crossover process are two new offspring. In this paper, the crossover process is based on a partially matched crossover (PMX) that was introduced by Lazzerini et.al with more advancement called MPMX [10]. Parents are taken from the existing chromosome $\left(P_{\text {keep }}\right)$. The pair of parents are randomly selected from the existing chromosomes $\left(P_{\text {keep }}\right)$. Then, three numbers are randomly selected. Let $a, b$, and $l$ represent the starting nodes for the first and the second chromosomes, and the length of the matching portion, respectively. For example: the chromosomes are $S_{A}$ and $S_{B}, l=4, a=3, b=7, n=10$.

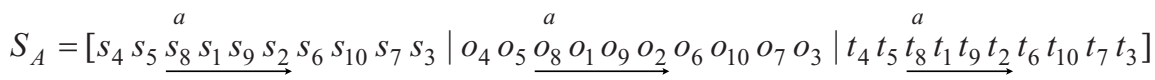

$$
\begin{aligned}
& S_{B}=\left[s_{7} s_{3} s_{2} s_{8} s_{5} s_{6} \stackrel{b}{\stackrel{s_{9} s_{1} s_{4} s_{10}}{\longrightarrow}}\left|o_{7} o_{3} o_{2} o_{8} o_{5} o_{6} \underset{o_{9} o_{1} o_{4} o_{10}}{\longrightarrow}\right| t_{7} t_{3} t_{2} t_{8} t_{5} t_{6} \underline{t_{9} t_{1} t_{4} t_{10}}\right]
\end{aligned}
$$

After the crossover

$$
\begin{aligned}
& C_{A}=\left[s_{2} s_{5} \stackrel{b}{s_{9} s_{1} s_{4} s_{10}} s_{6} s_{8} s_{7} s_{3}\left|o_{2} o_{5} \underline{o_{9} o_{1} o_{4} o_{10}} o_{6} o_{6} o_{8} o_{7} o_{3}\right| t_{2} t_{5} \underline{t_{9} t_{1} t_{4} t_{10}} t_{6} t_{8} t_{7} t_{3}\right] \\
& C_{B}=\left[s_{7} s_{3} s_{4} s_{10} s_{5} s_{6} \stackrel{s_{8} s_{1} s_{9} s_{2}}{\longrightarrow}\left|o_{7} o_{3} o_{4} o_{10} o_{5} o_{6} \underset{o_{8} o_{1} o_{9} o_{2}}{\longrightarrow}\right| t_{7} t_{3} t_{4} t_{10} t_{5} t_{6} \stackrel{a}{t_{8} t_{1} t_{9} t_{2}}\right]
\end{aligned}
$$

Then, the offspring $\left(C_{A}\right.$ and $\left.C_{B}\right)$ will fill the empty space in the population, replacing the disappearing chromosome $\left(P_{\text {disappear }}\right)$. A mutation operator is necessary to increase possibilities for getting better offspring. Mutation is held by modifying the chromosomes individually. The nodes are selected randomly. If generation has reached the cost function threshold, the generation should be stopped. The most optimal sequence for A/D task will be suggested to the operator. It is worth remarking that the optimal sequence is conducted based on the first part recognition algorithm chosen by the operator.

When provided with sufficient information, the system will generate the guidance path for the operator, which will guide the operator for the best part sequence of the maintenance task. In order to sense the guidance force, artificial and natural constraints are applied to the operator through the path. The fundamental idea of using the force field is that each object in virtual environment is surrounded by repulsive force field [7]. Since we already know the target position of our part position, the 
force field of whole environment can be calculated to assist the operator to reach the target position. We can prescribe the target position for aircraft parts. During the assembly task, target position will be the best position for each part in Cartesian coordinate related to the geometry constraint of the assembled parts. In disassembly task, target position is safe place (position) in Cartesian coordinate where we will put the part for the final position. The force applied for the guidance is a summation between the artificial and natural constraint. The following relation summarizes the force implementation [6] :

$$
\text { Constraint }_{\text {total }}(x, y, z)=\text { Constraint }_{\text {natural }}(y)+\text { Constraint }_{\text {artificial }}(x, z)
$$

The constraint along the plane $\mathrm{x}$ and $\mathrm{z}$ is used to guide the object to reach the prescribed target position, whereas the natural constraint is the constraint that naturally comes from the object. After one part has reached the target position, another guidance path for another part will be suggested to the operator. This process repeated until all part reaches their target positions. The sequence of task based on the results of the optimal sequence generation.

\section{Experiment and Result}

To implement optimal sequence generation, some experiments were conducted to test the algorithm. As shown in Fig. 3, An operator attempted to disassemble the assembled part. To accomplish the disassembly task, parameters such as gripper and direction of part were involved in cost value calculation. While in [5] the number of feasible sequence was involved, this trial did not involve that parameter in this experiment. Assume the possibility of escape part direction only limited to the $+\mathrm{z}$ and $-\mathrm{z}$ direction. The gripper was available in five types.

Table 1. Available Gripper

\begin{tabular}{c|l}
\hline Part & Available Gripper \\
\hline 1 & $\mathrm{G}_{1}, \mathrm{G}_{2}, \mathrm{G}_{3}$ \\
2 & $\mathrm{G}_{2}, \mathrm{G}_{3}, \mathrm{G}_{4}$ \\
3 & $\mathrm{G}_{1}, \mathrm{G}_{3}, \mathrm{G}_{4}$ \\
4 & $\mathrm{G}_{3}, \mathrm{G}_{4}, \mathrm{G}_{5}$ \\
5 & $\mathrm{G}_{3}, \mathrm{G}_{4}$ \\
6 & $\mathrm{G}_{5}$ \\
7 & $\mathrm{G}_{1}, \mathrm{G}_{2}, \mathrm{G}_{3}$ \\
8 & $\mathrm{G}_{2}, \mathrm{G}_{3}, \mathrm{G}_{4}, \mathrm{G}_{5}$ \\
9 & $\mathrm{G}_{1}, \mathrm{G}_{2}, \mathrm{G}_{3}, \mathrm{G}_{4}$ \\
\hline 10 & $\mathrm{G}_{5}$ \\
\hline
\end{tabular}

Initial population consisted of 100 individuals. The number of generation was limited to 50 generation. In every generation, maximum and average cost value was calculated. Below are the maximal cost value after 100 trial attempting. In every generation, maximum and average cost value was increased (Fig. 3) This indicate that 
solution is getting better from generation to generation. The final solution suggested for disassembly sequence are 10-8-6-2-5-9-1-4-7-3. Because the cost function did not involve a number of feasible sub-sequence and feasible sub-sequence modules, so there is a non-feasible sub-sequence in suggested solution.

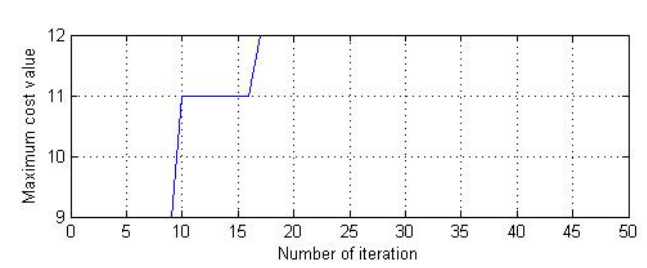

(a) Maximum cost function

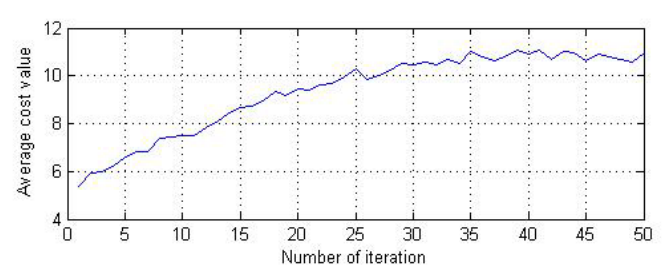

(c) Average cost function

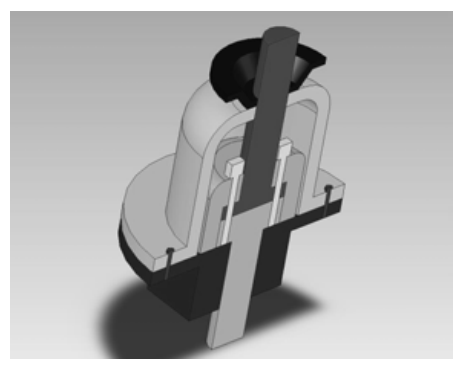

(b) Assembled part for experiment

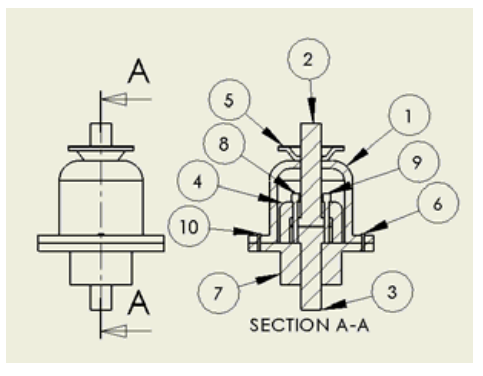

(d) Assembled part drawing for experiment

Fig. 3. Experiment result and picture of assembled part

\section{Conclusion}

In this paper, we have suggested the framework of the intelligent assembly/disassembly system for aircraft parts maintenance task. The algorithm and components of the system which deal with the complexity of the sequence selection by an optimalized method have been described in this paper. Some results have been showed refer to the selection of optimal sequence. In future works, sub-sequence module will be involved in the calculation of cost value to avoid non feasible subsequence appear in sequence solution. Furthermore, a novel algorithm and strategy in the haptic utilization has been developed.. In other words, this system could be a prototype of an efficient system for the purpose of aircraft part maintenance tasks. Since this is an ongoing research, further developments and inputs are desired to increase the effectiveness of the system. 


\section{Acknowledgement}

This work was supported by the Korea Research Foundation Grant funded by the Korean Government (MOEHRD) (KRF-2005-005-J09902) and was supported by NURI Project.

\section{References}

[1] http://www.sensable.com/. 4:23 pm. 26/12/2006

[2] Diego Borro, Joan Savall, Aiert Amundarain, Jorge Juan Gil, Alejandro Garcia-Alonso, Luis Matey, "A Large Haptic Device for Aircraft Engine Maintainability," IEEE Computer Graphics and Applications, vol. 24, no. 6, pp. 70-74, Nov/Dec 2004.

[3] Joan Savall, Diego Borro, Jorge Juan Gil, Luis Matey, "Description of a Haptic System for Virtual Maintainability in Aeronautics," IEEE International Conference on Intelligent Robots and Systems, Laussane, Switzerland, October 2002.

[4] Alex B. Kuang, Shahram Payandeh, Bin Zheng, Frank Henigman, Christine L. MacKenzie, "Assembling Virtual Fixtures for Guidance in Training Environments," haptics, pp. 367-374, 12th International Symposium on Haptic Interfaces for Virtual Environment and Teleoperator Systems (HAPTICS'04), 2004.

[5] L. M. Galantucci; G. Percoco \& R. Spina, "Assembly and Disassembly Planning by using Fuzzy Logic \& Genetic Algorithms," International Journal of Advanced Robotic Systems, volume 1 Number , 2004

[6] Daniel Galeano and Shahram Payandeh, "Artificial and Natural Force Constraints in Haptic-aided Path Planning," IEEE International Workshop on Haptic Audio Visual Environments and their Applications, Ottawa, Canada, 1-2 October 2005

[7] Dongbo Xiao and Roger Hubbold. "Navigation Guided by Artificial Force Fields," Proceedings of ACM CHI 98 Conference on Human Factors in Computing Systems, vol. 1, pp 179-186. ACM SIGCHI, Addison Wesley, April 1998

[8] "Open Inventor 6", Mercury Computer System, 2006

[9] Randy L. Haupt and Sue Ellen Haupt, "Practical Genetic Algorithms 2nd Edition,” John Willey and Sons, USA, 2004

[10] Lazzerini B., Marcelloni F., "A Genetic Algorithm for generating Optimal Assembly Plans", Artificial Intelligence in Engineering, Vol. 14, pp. 319-329, 2000 